\title{
Isolation of monomeric photosystem II that retains the subunit PsbS
}

\author{
Patrycja Haniewicz • Daniele De Sanctis • Claudia Büchel • \\ Wolfgang P. Schröder • Maria Cecilia Loi - Thomas Kieselbach • \\ Matthias Bochtler • Dario Piano
}

Received: 22 December 2012/ Accepted: 7 August 2013/Published online: 24 August 2013

(C) The Author(s) 2013. This article is published with open access at Springerlink.com

\begin{abstract}
Photosystem II has been purified from a transplastomic strain of Nicotiana tabacum according to two different protocols. Using the procedure described in Piano et al. (Photosynth Res 106:221-226, 2010) it was possible to isolate highly active PSII composed of monomers and dimers but depleted in their PsbS protein content. A "milder" procedure than the protocol reported by Fey et al. (Biochim Biophys Acta 1777:1501-1509, 2008) led to almost exclusively monomeric PSII complexes which in part still bind the PsbS protein. This finding might support a role for PSII monomers in higher plants.
\end{abstract}

P. Haniewicz $\cdot$ M. Bochtler $\cdot$ D. Piano

International Institute of Molecular and Cell Biology,

Ul. Ks. Trojdena 4, 02109 Warsaw, Poland

D. De Sanctis - D. Piano

European Synchrotron Radiation Facility,

Rue Jules Horowitz 6, 38000 Grenoble, France

C. Büchel

Institute of Molecular Biosciences, University of Frankfurt, Max von Laue Straße 9, 60438 Frankfurt am Main, Germany

W. P. Schröder · T. Kieselbach

Umeå Plant Science Center and Institute of Chemistry,

Linnaeus väg 10, 90187 Umeå, Sweden

M. C. Loi · D. Piano $(\square)$

Department of Life and Environmental Sciences,

University of Cagliari, V.le S. Ingnazio da Laconi 13,

09123 Cagliari, Italy

e-mail: dario.piano@unica.it

M. Bochtler

Department of Bioinformatics, Institute of Biochemistry

and Biophysics, Pawinskiego 5a, 02-106 Warsaw, Poland
Keywords Photosystem II · Photosynthesis · PsbS · Thylakoid membranes - Nicotiana tabacum .

Oligomeric state

\section{Introduction}

Photosystem II (PSII) catalyzes the first light-dependent reaction in oxygenic photosynthesis, the splitting of water molecules into molecular oxygen, protons, and electrons. The proton gradient across the thylakoid membrane then drives the ATP synthesis, while electrons are transferred to plastoquinone and eventually converted to reducing equivalents (Cardona et al. 2012).

PSII seems to occur in both monomeric and dimeric states in vivo. PSII monomers have been associated with the physiological turnover of the dimeric state: typically dimers renew via monomerization and subsequent exchange of the D1 protein, an important polypeptide involved in the process of charge separation and electron transport (Pokorska et al. 2009). Other studies have also suggested that the PSII oligomeric state is dependent on localization. Dimers are reported to occur in thylakoid grana while monomers are predominant in stromal lamellae. Within this distribution, the PSII dimers are considered to be active in oxygen evolution, in contrast to monomers, that are generally less active and heterogeneous (Danielsson et al. 2006).

The PsbS subunit of PSII is considered to be a crucial component in the regulation of the PSII photochemistry, because PsbS mutants are defective in non-photochemical quenching ( $\mathrm{Li}$ et al. 2000). In contrast to photochemical quenching, which describes the de-excitation of PSII with concomitant electron transport, non-photochemical quenching describes the reduction of PSII fluorescence due to the production of heat (Niyogi et al. 2005). 
Non-photochemical quenching is controlled by $\mathrm{pH}$ in the thylakoid lumen, which has been hypothesized to be sensed by the PsbS protein (Szabó et al. 2005). However, it is not clear how PsbS might mediate the switching of PSII between a fully active state and a protective state of reduced activity induced by the intense light. Prior to the isolation of the PsbS mutant, the xanthophyll cycle was pinpointed as a key player in non-photochemical quenching. Several possible modes of action of the PsbS protein are currently discussed. First, the PsbS protein might influence the xanthophyll cycle (Szabó et al. 2005). Second, the PsbS protein could interact directly with the PSII core (Li et al. 2004; Kiss et al. 2008). Finally, and perhaps more plausibly, the PsbS protein could affect the conformation of the light harvesting complex II (LHCII) (Horton et al. 2005).

Here we report a "milder" extraction of PSII from Nicotiana tabacum, which resulted in samples constituted mainly of monomeric PSII complexes divided in two populations one of which binds the PsbS protein. This raises the question in which form the functional PSII is organized in vivo in higher plants.

\section{Results}

\section{Oligomeric state of PSII preparations}

PSII was isolated from $N$. tabacum plants that had been genetically modified to express the protein subunit PsbE with a hexahistidine tag as described earlier (Fey et al. 2008). Leafs were harvested $5 \mathrm{~h}$ before the onset of the light period and PSII complexes were isolated either according to a previously published protocol (Piano et al. 2010, protocol A) or to a new modified "milder" protocol (protocol B), which is based on Fey et al. 2008. In the new method (protocol B) the detergent to chlorophyll ratio was reduced to half and glycerol was included in all buffers. These small alterations had a major effect on the behavior of PSII during purification. In the first chromatography purification step with a Ni-NTA resin, we noted that PSII prepared according to protocol B tended to elute slightly earlier (at lower imidazole concentration) than when using the protocol A suggesting PSII complexes of different subunit composition or alternatively a different monomer to dimer ratio (Fig. 1a). The latter hypothesis was tested by Blue-Native gel electrophoresis (BN-PAGE) confirming that PSII extracted using protocol B migrates mainly in a single band at an apparent molecular mass of $340 \mathrm{kDa}$ representing the monomeric PSII, accompanied by only little amounts of dimers (band migrating at an apparent mass of $680 \mathrm{kDa}$ ) (Fig. 2). In contrast, when protocol A was used, several bands were observed, corresponding to the monomer, dimer, and smaller incomplete complexes
(Fig. 2). A further step of purification by size exclusion chromatography confirmed the results shown in Fig. 2. In case of PSII extracted with protocol B, a single very sharp peak was observed (Fig. 1b). In contrast, protocol A led to two overlapping peaks, which reflect the presence of different species (Fig. 1b and inset Fig. 1c). The two separated oligomeric forms were found to be very stable over time. Thus, when monomeric or dimeric PSII obtained using protocol A and enriched by size exclusion chromatography were re-injected, they migrated according to the same elution profile, indicating that exchange between monomers and dimers was very slow, if it occurred at all (Fig. 1c) and that the complexes were very stable.

Based on those findings, we used BN-PAGE to analyze the thylakoids solubilized according to protocol A or B. These thylakoids showed different but reproducible separation patterns depending on the solubilization protocol (Fig. 1d). Western blots on second dimension SDS-PAGE helped to identify the main constituents and also to estimate the ratio between PSII monomers and dimers. From those experiments the absence of dimeric PSII in thylakoids prepared according to protocol B was evident by the absence of any anti-D1 signal at the respective mass, whereas when using the harsher protocol A, D1 could be detected for both monomeric and dimeric PSII (Fig. 1d). As observed in other reports, in both cases the D1 signal resulted in two pools of spots equivalent to D1 monomers and D1 aggregates that migrate at almost double of the expected mass (Ishikawa et al. 1999). In order to test whether the results observed were only related to the His-tag present in the transplastomic strain, the same procedure was carried out using wild-type tobacco plants. Those experiments revealed the same solubilization patterns (data not shown).

In order to define whether those results were somehow representative of the composition of the thylakoid membrane, we calculated the yield for both preparations. With the harsher protocol A, all thylakoids (35 mg of total chlorophylls) were solubilized with a final harvest of $0.5 \mathrm{mg}$ of PSII chlorophylls, i.e., a yield of about $1.4 \%$. On the contrary, with the milder protocol B starting from the same amount of thylakoids only $20 \mathrm{mg}$ of chlorophylls went in solution, i.e., only about $60 \%$ of $\mathrm{Chl}$ was recovered. However, from those $20 \mathrm{mg}$ the final amount of PSII chlorophylls harvested was typically $0.4 \mathrm{mg}$, implying an yield of $2 \%$ of solubilized material or $1.1 \%$ of total Chl. This value is comparable with the recovery observed in protocol $\mathrm{A}$ and indicates that the PSII monomeric form is present in roughly the expected amounts judging from total chlorophylls.

Subunit composition of the two PSII preparations

The two PSII purified batches were next investigated for their subunit composition by denaturing gel electrophoresis and mass spectrometry. The main PSII core subunits were 
Fig. 1 a Elution profile recoded at $280 \mathrm{~nm}$ of the NiNTA affinity chromatography for the samples prepared according to protocol $\mathrm{A}$ (dashed lines) and B (dotted lines), respectively. b Size exclusion chromatography of the PSII preparations. The elution profile of the sample prepared according to the protocol A (PSII-A) is shown in gray and the profile of the sample prepared according to the protocol B (PSII-B) is depicted in black. Profiles were recorded at $280 \mathrm{~nm}$ (dotted lines) and $664 \mathrm{~nm}$ (dashed lines). c Size exclusion chromatography recorded at $280 \mathrm{~nm}$ (dotted lines) and $664 \mathrm{~nm}$ (dashed lines) of the monomer (black) and dimer (gray) enriched fractions collected after a previous step of size exclusion chromatography (b PSII-A, gray profile). Elution fractions compositions of the two pools used for these experiments were analyzed by BN-PAGE (inset). The boxes in the inset indicate the two pools collected for the runs. d BNPAGE of thylakoids (T, $8 \mu \mathrm{g}$ Chl) solubilized according to protocol A (on the left) or protocol B (on the right). The lanes labeled with PSII show the correspondent PSII samples ( $8 \mu \mathrm{g} \mathrm{Chl})$, used as a reference. The boxes labeled with anti-D1 represent the western blots for the D1 subunit in the thylakoids after 2nd dimension SDSPAGE, whereas below the second dimension SDS-PAGES are shown
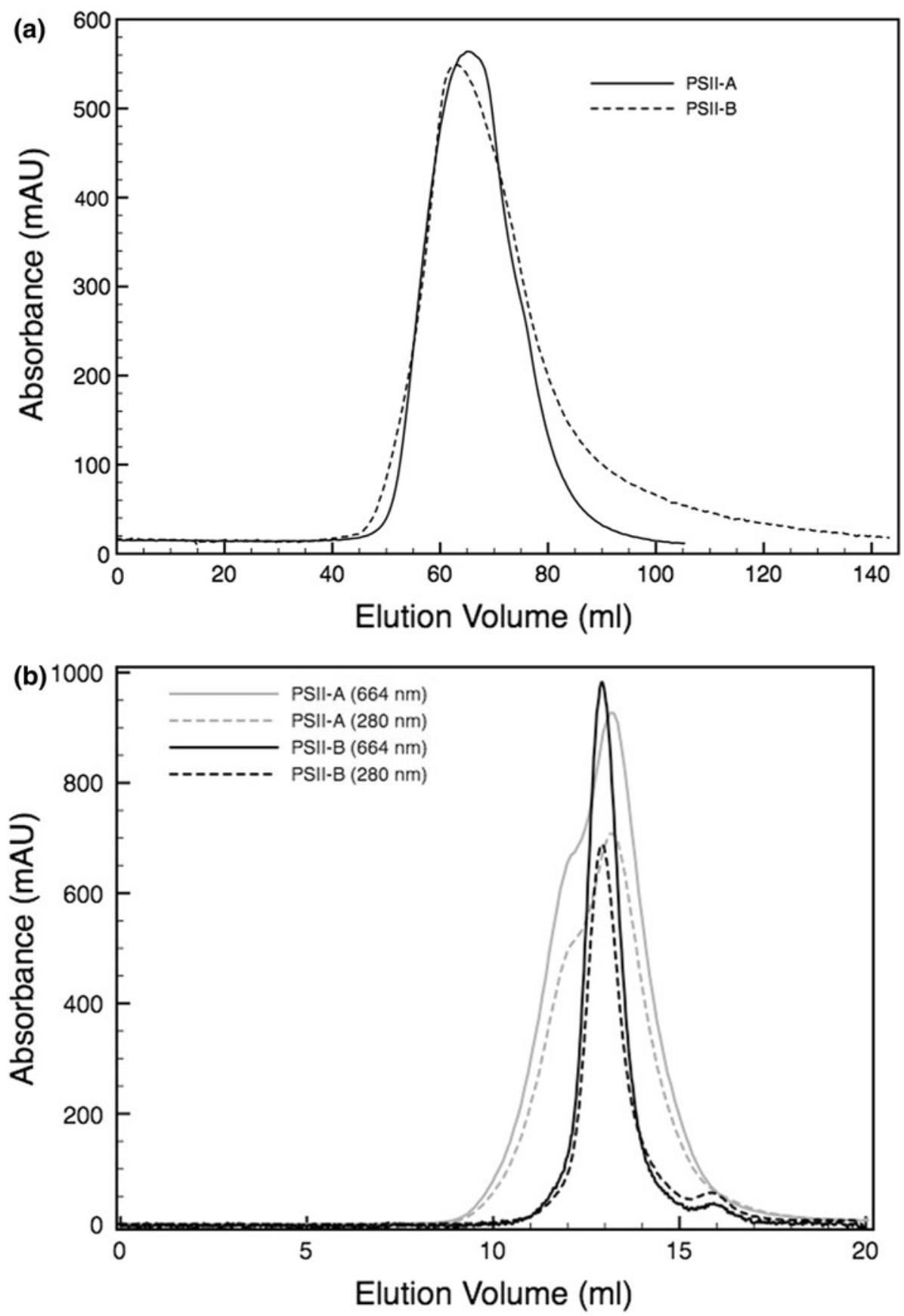

present in both preparations. However, the samples obtained with protocol B contained the PsbS subunit that was totally absent or only present in trace amounts in samples from protocol A, as shown in Fig. 3.

Further investigation by mass spectrometry (Table 1) shows that protocol A retained four $\mathrm{CAB}$ proteins (CAB2, CAB25, CAB26, CAB36). Both preparations contained significant amounts of the subunit CP29 (product of the gene Lhcb4), but none of the major LHCII (polypeptides Lchb1-3). Western Blotting using commercially available polyclonal antibodies confirmed the correct assignment of the different subunits (Table 1). These experiments show that the PsbS protein is present in much higher abundance in B than A samples and that the major LHCII are missing in both preparations. Based on these findings, we will refer to the dimeric fraction obtained from protocol A as PSIId, the monomeric fraction as PSIIm and the monomeric fraction, enriched in PsbS obtained from protocol B as PSIImM (where M stand for Mild). Western blots on the BN-PAGE and on its second dimension SDS-PAGE were performed in order to check whether the presence of PsbS in the PSIImM samples was actually due to the binding, or 


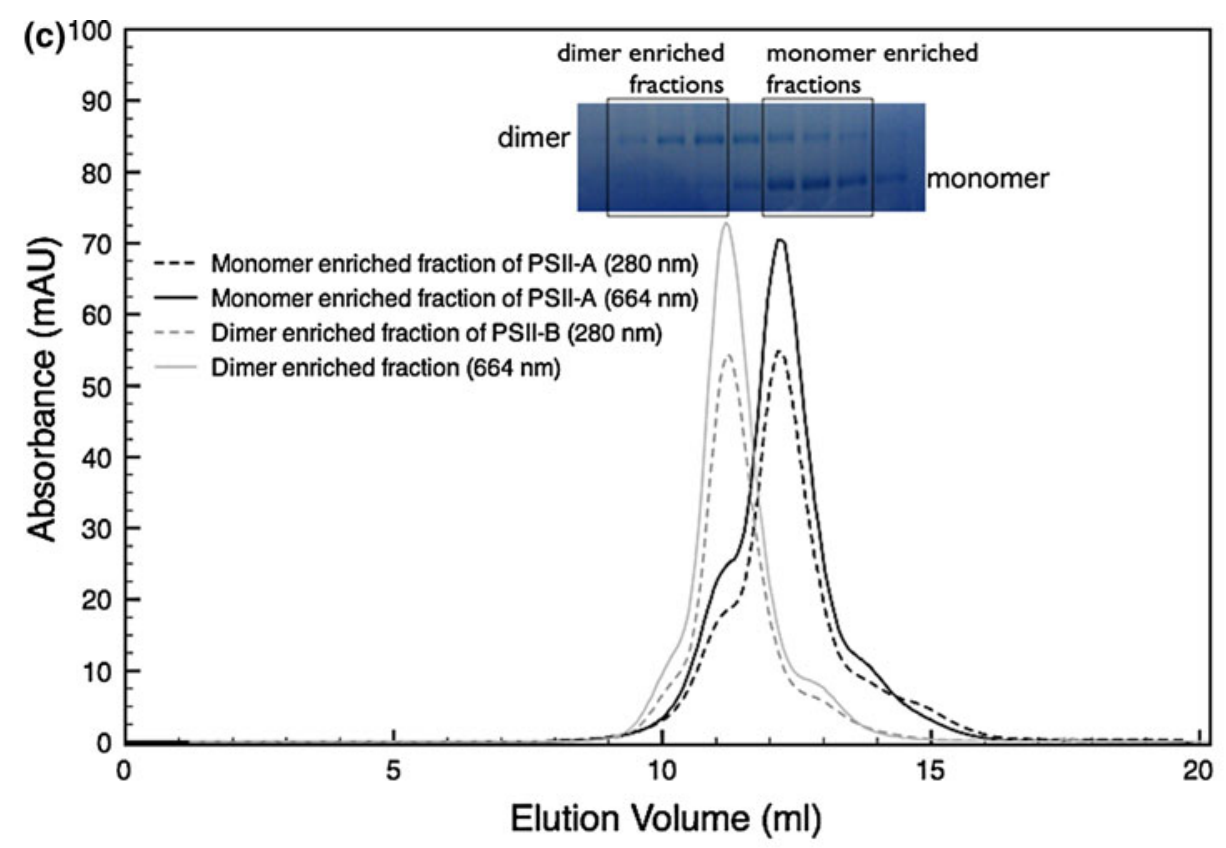

(d)

Protocol A
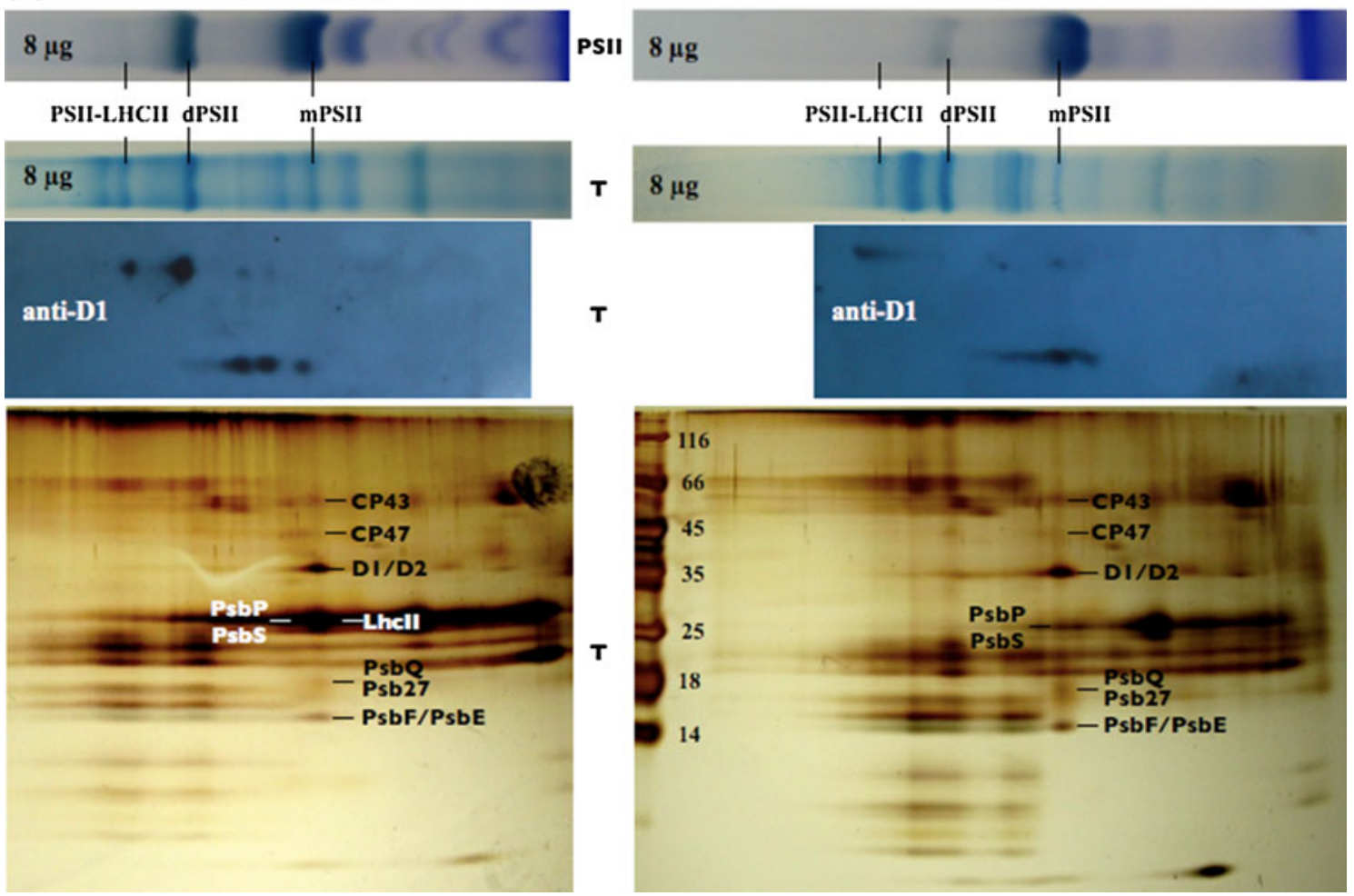

14

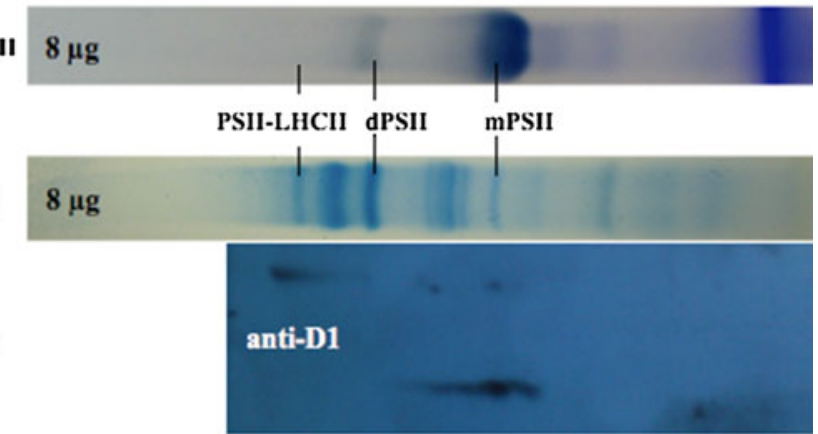

Fig. 1 continued

if it was just the result of a co-migration with PSII monomers. In both cases an anti-PsbS reaction was only observed at the level of PSII monomers, neither in dimers nor as a single PsbS protein. However, when performing BN-PAGE followed by western blotting on thylakoids obtained by protocol $\mathrm{B}$, diffuse signals starting from masses of $360 \mathrm{kDa}$ until $20 \mathrm{kDa}$ were obvious (data not shown). Moreover, we observed also that the single-band obtained from the BN-PAGE on PSIImM samples appeared composite when resolved in second dimension SDS-PAGE (Fig. 2c). This fact suggested that the PSIImM preparation consists of two monomeric PSII populations. 


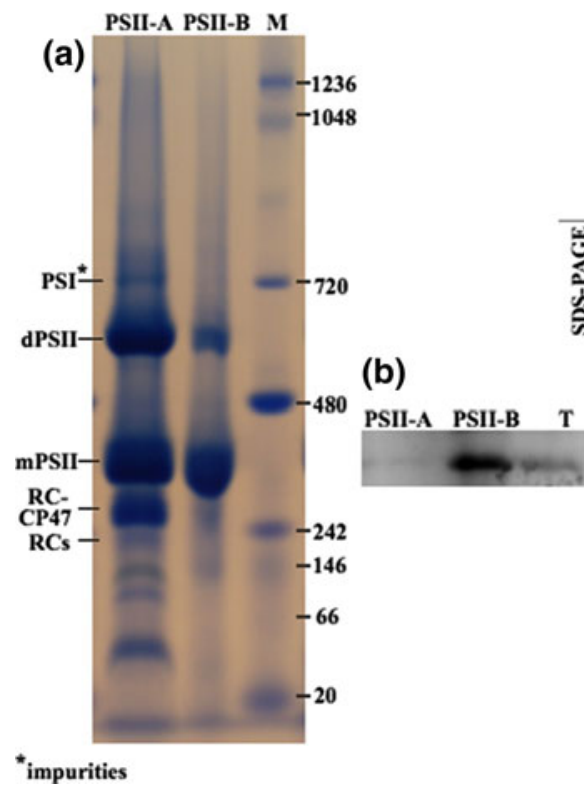

(c)

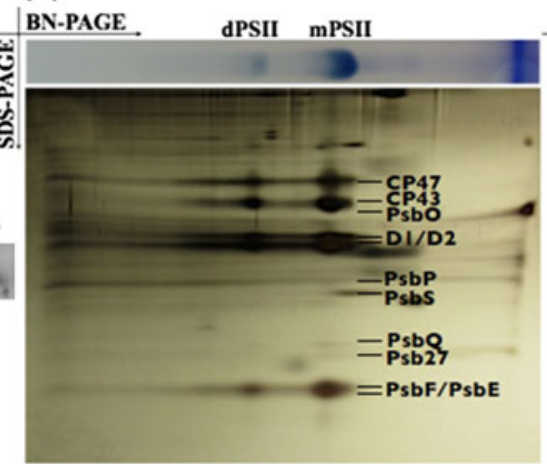

(d)

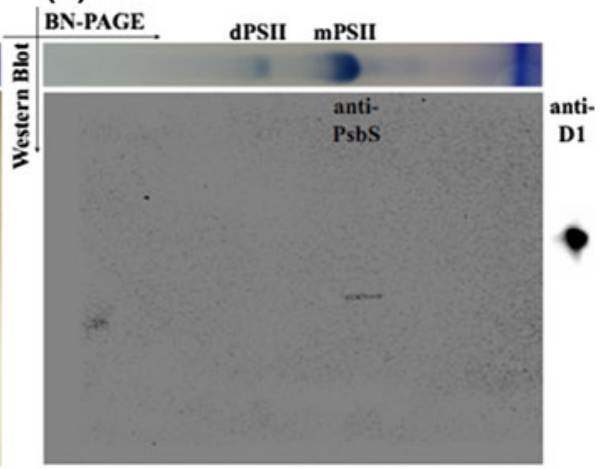

Fig. 2 On the left side the BN-PAGE of samples obtained with protocol A (lane PSII-A) and protocol B (lane PSII-B) is shown (a), the lane $M$ indicates the standard. The associated western blotting reaction using anti-PsbS for the samples PSII-A, PSII-B, and the thylakoids (T) at the level of the PSII monomers is also shown (b).

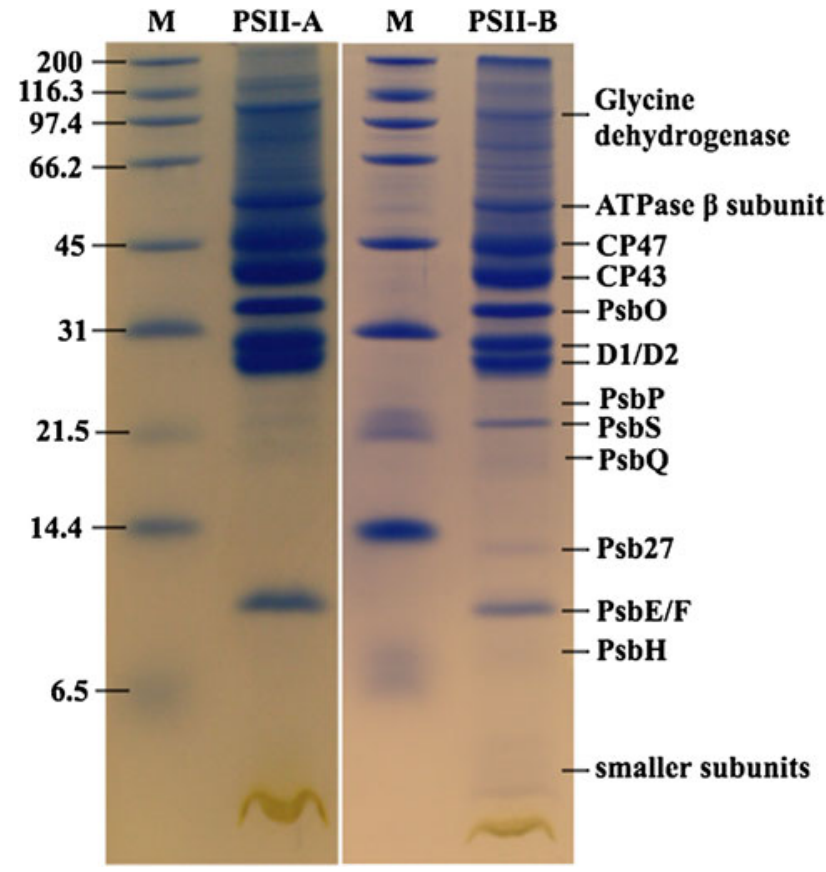

Fig. 3 Denaturing SDS-PAGE analysis of PSII preparations according to protocol A (PSII-A) and protocol B (PSII-B). Lane $M$ shows the molecular marker. The labels for protein bands represent the identifications as found by ESI LC-MS/MS peptide mass finger printing (see Table 1)

Of those two populations the lighter one showed a PsbS band while interestingly the PsbO band was missing (Fig. 2c, d). On the contrary, the PSIImM fraction not able
Loading was equivalent to $5.1 \mu \mathrm{g}$ Chl for PSII-A and $3.2 \mu \mathrm{g}$ Chl for PSII-B. On the center-right the second dimension SDS-PAGE obtained after a BN-PAGE of PSII-B as a first dimension is shown (c). On the right the western blots for anti-PsbS (from the whole gel) and anti-D1 (from the lane of monomers) are depicted (d)

to bind PsbS showed a typical PsbO band (Fig. 2c), suggesting that only one fraction of the total monomers were able to bind PsbS in the PSIImM samples (Fig. 2d). Thus, in the thylakoid membrane PsbS is found in different forms and associations, but especially the results from the second dimension SDS-PAGE provide a strong indication of a specific binding of PsbS to monomeric PSII (Fig. 2).

\section{Rates of oxygen evolution of the PSII preparations}

In order to analyze if the isolated fractions were functionally active we measured the oxygen evolution of the PSIIm, PSIId, and PSIImM samples as well as of both samples obtained after the first purification step (NiNTA elution from protocols A and B). As PSIIm and PSIId are stable and their oligomeric state is not exchanged over time, we could independently determine their activities observing for both high rates of oxygen evolution (Table 2). Surprisingly in the milder extraction, yielding mainly monomeric PSII, only low rates of oxygen evolution $\left(58 \mu \mathrm{mol} \mathrm{O} \mathrm{O}_{2} / \mathrm{mg} \mathrm{chl} \mathrm{h}\right)$ were observed indicating a much lower activity for the PSIImM sample compared to the PSIIm sample (Table 2).

\section{Spectroscopy of the two PSII preparations}

Absorption spectra for the PSIIm and PSIId fractions and for the PSIImM sample were recorded in the wavelength range between 370 and $750 \mathrm{~nm}$ and normalized to their $\mathrm{Q}_{\mathrm{y}}$ 
Table 1 Subunit composition of PSII-A and PSII-B analysed by ESI LC-MS/MS peptide mass finger printing (MS) and western blots in comparison to thylakoids (Thyl). For western blots equal amounts of Chl were load

\begin{tabular}{|c|c|c|c|c|c|}
\hline \multirow{2}{*}{$\begin{array}{c}\text { Protein } \\
\text { PSII }\end{array}$} & \multicolumn{2}{|c|}{$M S^{*}$} & \multicolumn{3}{|c|}{ Western Blot } \\
\hline & $P S I I-A$ & PSII-B & PSII-A & PSII-B & Thyl. \\
\hline PsbA (D1) & + & + & - & $=0$ & 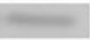 \\
\hline PsbB (CP47) & + & + & 2 & 2 & $=$ \\
\hline PsbC (CP43) & + & + & $=$ & $=$ & 3 \\
\hline PsbD (D2) & + & + & & n.d. ** & \\
\hline PsbE & + & + & & n.d. ** & \\
\hline PsbF & + & + & & n.d. ** & \\
\hline PsbH & + & + & & n.d.** & \\
\hline PsbL & + & + & & n.d. ** & \\
\hline Psbo & + & + & $=$ & - & - \\
\hline PsbP & + & + & & $=$ & \\
\hline PsbQ & + & + & & 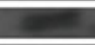 & \\
\hline PsbJ & - & + & & n.d. ** & \\
\hline Psb27 & + & + & & n.d. ** & \\
\hline Lhcb1 & - & - & \begin{tabular}{|l} 
\\
\end{tabular} & 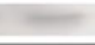 & $\longrightarrow$ \\
\hline Lhcb4 & + & + & $\square$ & $=$ & $=$ \\
\hline Lhcb5 & - & - & & 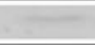 & $=$ \\
\hline PsbS & + & + & & 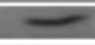 & - \\
\hline CAB2 & + & - & & n.d. ** & \\
\hline CAB25 & + & - & & n.d.** & \\
\hline CAB26 & + & - & & n.d.** & \\
\hline CAB36 & + & - & & n.d. ** & \\
\hline \multicolumn{6}{|c|}{ Non PSII proteins } \\
\hline Glycine dehydrogenase & + & + & & n.d. ** & \\
\hline $\begin{array}{l}\beta \text {-subunit of mitochondrial } \\
\text { ATPase }\end{array}$ & + & + & & n.d. ** & \\
\hline
\end{tabular}

absorption maximum to facilitate their comparison (Fig. 4). Generally, the three spectra showed a comparable absorption profile regarding the $\mathrm{Q}_{\mathrm{x}}$ and the $\mathrm{Q}_{\mathrm{y}}$ regions. However, the intensities differed significantly in the wavelength range between 450 and $520 \mathrm{~nm}$. In this region the absorbance intensity was the lowest for the monomeric PSIImM, followed by PSIId and finally PSIIm. Furthermore, difference spectra between PSIImM and PSIIm feature several characteristic bands. In particular the absorbance at 470 and $490 \mathrm{~nm}$ is enhanced in PSIIm, accompanied by minor changes in the $\mathrm{Chl} b$ and $\mathrm{Chl} \mathrm{a} \mathrm{Q}_{\mathrm{y}}$ region (Fig. 4 inset). These differences indicate the presence of carotenoids, probably associated to some light harvesting proteins in PSIIm, in agreement with the detection of four $\mathrm{CAB}$ proteins and the CP29 protein by mass-spectroscopy.

\section{Discussion}

Most PSII preparations described in the literature contain dimers (Boekema et al. 1995; Dekker and Boekema 2005). However, recently a monomeric form in vivo has been reported (Takahashi et al. 2009; Watanabe et al. 2009; Pagliano et al. 2011). Different oligomeric states of PSII have been associated with different locations in thylakoid membranes (Danielsson et al. 2006). Dimers are found mainly in the grana, together with PSII supercomplexes that consist of dimers associated with antenna proteins (see Fig. 5; Table 4 in Danielsson et al. 2006). PSII monomers are located mainly in the margins of the grana, in the stroma lamellae and in the distal region of the stroma lamellae, the so-called Y100 region. Immunogold labeling experiments performed on maize thylakoids using antibodies against PsbS have shown that PsbS tends to be associated to stroma lamellae in leaves exposed to an intermediate or intense light regime (Teardo et al. 2007) similar to the one used in this work. However, some reports have also shown PsbS strongly associated to the grana (Kiss et al. 2008; Horton et al. 2008; Kereïche et al. 2010) suggesting an ubiquitous localization of this protein in thylakoid membranes.

We suspect that the "milder" PSII purification protocol B reported here solubilizes only monomeric PSII present in the stroma, while the "harsher" protocol solubilizes also PSII from the internal grana cores. As shown in Fig. 1d, the thylakoids solubilized following the two different protocols present different patterns. In particular from western blots analysis using anti-D1 the milder protocol seems to contain only PSII monomers and some weak signal at higher molecular weight due to traces of PSII-LHCII supercomplexes; on the contrary in the harsher protocol the signals are most pronounced at the level of the PSII dimers. According to this interpretation, PSIId could be considered of grana origin, whereas PSIIm would represent an

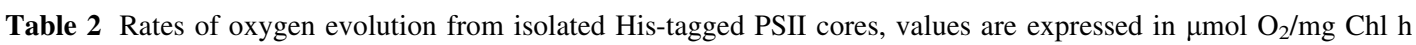

\begin{tabular}{lllc}
\hline Preparation & Chromatography step & & \\
\cline { 2 - 4 } & NiNTA & S.E.C. & 2nd pool \\
\cline { 2 - 4 } & Single pool & 1 st pool & $544 \pm 31$ (enriched PSIIm) \\
\hline PSII-A & $826 \pm 23$ (PSIId, PSIIm, RC-CP47, RC) & $1100 \pm 22$ (enriched PSIId) & $58 \pm 5$ (PSIImM) \\
PSII-B & $71 \pm 4$ (PSIImM, PSIId in traces) & - &
\end{tabular}

Values represent means \pm standard deviations of 3 independent measurements from the same preparation 


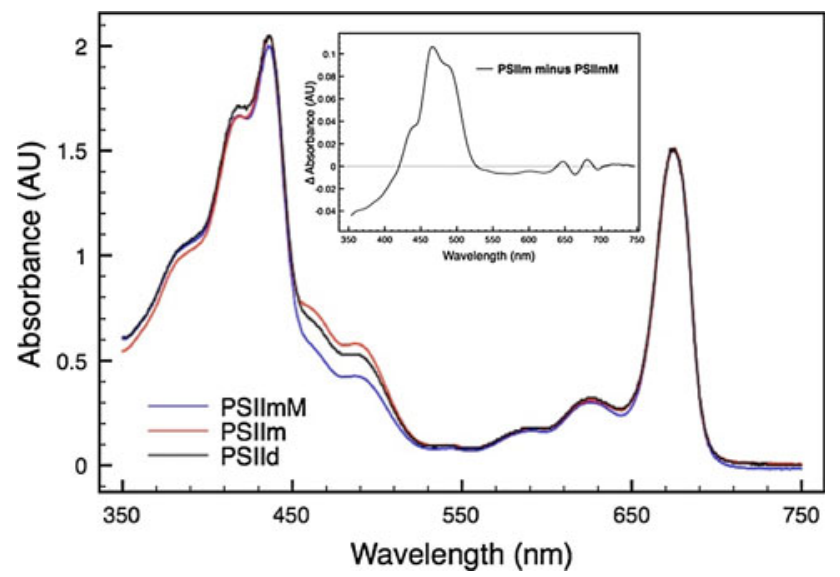

Fig. 4 Absorption spectra of the PSIIm (red line) and the PSIId (black line) from the preparation A and of the PSIImM (blue line) from the preparation B. The inset shows difference spectrum between monomers (PSIIm minus PSIImM)

enrichment of PSII of lamellar origin. The presence and (near) absence of PsbS in our two samples would then reflect the physiological association with PSII, i.e., PsbS would be preferentially attached to stromal PSII (PSIImM). This is still in line with the observations by Fey et al. (2008), where PsbS was also reported to be present in PSII cores. In those preparations probably all PSII complexes were isolated, and as in our PSII-A the PsbS content was relatively low. The composite constitution of the PSIImM samples (Fig. 2c) is due to the presence of two sub-populations of monomeric PSII in which one of them contains PsbS and lacks PsbO. As PsbO is important for the stabilization of the oxygen evolving center (Yi et al. 2005), this fraction can be expected to be non-functional on the one hand and highly sensitive to photo-damage on the other hand. Hence, the presence of PsbS in the PsbO deficient population is mechanistically reasonable. This sub-population of PSII monomers is probably similar to the lamellar PsbO-deficient PSII particles observed by Bassi et al. (1995) and to the inactive monomeric PSII present in the Y-100 domain reported by Danielsson et al. (2006). Finally, the other sub-population of PSIImM that contains PsbO, but lacks PsbS could originate from the stromalamellae domain. This assignment would agree with previous observations of a partially active PSII monomer in this region of the membranes (Danielsson et al. 2006).

\section{Materials and methods}

Growth and cultivation of tobacco plants

The transplastomic plants of $N$. tabacum, that carry a hexahistidine tag sequence at the $5^{\prime}$ end of the gene coding for the PsbE subunit, were described by Fey et al. (2008). The plants were kept at a constant temperature of $25{ }^{\circ} \mathrm{C}$ at $50 \%$ relative humidity and grown for 10-12 weeks under a light regime of $12 \mathrm{~h} /$ day, with a light intensity of 150-200 $\mu \mathrm{mol}$ photons/( $\left.\mathrm{s} \mathrm{m}^{2}\right)$.

\section{Thylakoid preparation}

Thylakoid membranes were purified as reported previously by Fey et al. (2008) with only minimal modifications in the solubilization step. In brief, thylakoids were resuspended in $20 \mathrm{mM}$ MES-NaOH, pH 6.5; $100 \mathrm{mM} \mathrm{NaCl} ; 5 \mathrm{mM}$ $\mathrm{MgCl}_{2} ; 10 \mathrm{mM} \mathrm{NaHCO} 3 ; 12.5 \%$ (v/v) glycerol prior solubilization. PSIImM core complexes were obtained from thylakoids membranes solubilized for $5^{\prime}$ at $4{ }^{\circ} \mathrm{C}$ at a final chlorophyll concentration of $3 \mathrm{mg} / \mathrm{ml}$ (protocol B). The PSII core complex lacking of PsbS (protocol A) was prepared starting from thylakoids membranes solubilized for $15^{\prime}$ at $4{ }^{\circ} \mathrm{C}$ at a final concentration of $1 \mathrm{mg} / \mathrm{ml}$ chlorophyll. In both cases solubilization was carried out using $20 \mathrm{mM}$ $\beta$-dodecylmaltoside ( $\beta$-DDM).

PSII core complex purification by affinity chromatography

Photosystem II samples were prepared using $\mathrm{Ni}$ affinity chromatography. PSII isolated following the protocol A was prepared according to Piano et al. (2010); PSII isolated following the protocol B was prepared according to Fey et al. (2008) with minor changes. In brief, for the protocol A the washing buffer was free of glycerol (20 mM MES$\mathrm{NaOH}, \mathrm{pH} 6.5 ; 100 \mathrm{mM} \mathrm{NaCl} ; 10 \mathrm{mM} \mathrm{NaHCO} 3 ; 15 \mathrm{mM}$ imidazole; $1 \mathrm{M}$ betaine). For protocol $\mathrm{B}$ the washing buffer consisted of $20 \mathrm{mM}$ MES-NaOH, pH $6.5,100 \mathrm{mM} \mathrm{NaCl}$, $10 \mathrm{mM} \mathrm{NaHCO} 3,15 \mathrm{mM}$ imidazole, $1 \mathrm{M}$ betaine, $12.5 \%$ (v/v) glycerol. In both cases PSII cores were then eluted using $40 \mathrm{mM}$ MES-NaOH, pH 6.5; $20 \mathrm{mM} \mathrm{NaCl} ; 5 \mathrm{mM}$ $\mathrm{MgCl}_{2} ; 1 \mathrm{mM} \mathrm{CaCl} ; 10 \mathrm{mM} \mathrm{NaHCO}{ }_{3} ; 300 \mathrm{mM}$ imidazole; $1 \mathrm{M}$ betaine. In both preparations the washing and the elution buffers contained $0.02 \%$ instead of $0.03 \%(\mathrm{w} / \mathrm{v})$ $\beta$-DDM. The volumes of washing were increased to $12 \mathrm{CV}$.

\section{Size exclusion chromatography}

Both preparations were concentrated using Vivaspin 20 ultrafiltration membranes with $100 \mathrm{kDa}$ cutoff until a final volume of $500 \mu \mathrm{l}$. The protein sample was loaded on a gel filtration column (Superose 6 10/300 GL, GE Healthcare) equilibrated with gel filtration buffer (40 mM MES-NaOH, pH 6.5; $20 \mathrm{mM} \mathrm{NaCl} ; 5 \mathrm{mM} \mathrm{MgCl} 2 ; 1 \mathrm{mM} \mathrm{CaCl}_{2} ; 10 \mathrm{mM}$ $\mathrm{NaHCO}_{3} ; 0.02 \%$ (w/v) $\beta$-DDM). The main peaks were pooled and concentrated by ultrafiltration (Vivaspin 20, 
$100 \mathrm{kDa}$ cutoff) to a volume of $200 \mu \mathrm{l}$ and when necessary re-injected for a second separation.

\section{Absorption spectroscopy and chlorophyll determination}

Thylakoid protein content was measured referring to the $\mathrm{Chl} \mathrm{a}$ and $\mathrm{Chl} \mathrm{b}$ concentrations. The analysis was done photometrically in $80 \%(\mathrm{v} / \mathrm{v})$ acetone using a Pharmacia Biotech Ultrospec 4000 spectrophotometer and Chl concentrations were calculated according to Porra et al. (1989). Absorption spectra were recorded at room temperature in the range of 370-750 nm with an optical path length of $1 \mathrm{~cm}$ and a band-pass of $2 \mathrm{~nm}$.

Polyacrylamide gel electrophoresis and western blots

For denaturing SDS PAGE, $10 \%(\mathrm{w} / \mathrm{v})$ separating polyacrylamide/urea gels with $4 \%(\mathrm{w} / \mathrm{v})$ stacking gels were used (Schägger and Jagow 1987). Samples were denatured with Rotiload (Roth) at room temperature before loading, and after the electrophoretic separation the gels were stained with Coomassie brilliant blue G250. Blue native gel electrophoresis was carried out using 3-12\% (w/v) continuous gradient gels according to Schägger and Jagow 1991. PSII complexes at $0.2 \mathrm{mg} \mathrm{Chl} / \mathrm{ml}$ were mixed with 0.25 volumes of Coomassie Blue Solution (5\% (v/v) serva Blue G, $750 \mathrm{mM}$ aminocaproic acid, $35 \%$ (w/v) sucrose). Electrophoresis was carried out at $205 \mathrm{~V}$ for $5 \mathrm{~h}$ at $4{ }^{\circ} \mathrm{C}$. For 2D separation, the strips from the BN-PAGE were excised and denaturated with Rotiload (Roth) at room temperature for $20 \mathrm{~min}$. After denaturation the strips were placed on the top of a denaturing SDS-PAGE as described above and sealed with Agarose $0.5 \%$ in cathode buffer. For Western blots, gels were first equilibrated in cathode buffer (25 mM Tris/HCl, pH 9.4; 40 mM glycine; $10 \%$ (v/ v) methanol). For transfer of the proteins onto a PVDF membrane, filter papers soaked in two different anode buffers $(0.3 \mathrm{M}$ Tris/ $\mathrm{HCl}, \mathrm{pH} 10.4 ; 10 \%(\mathrm{v} / \mathrm{v})$ methanol and $25 \mathrm{mM}$ Tris/ $\mathrm{HCl}, \mathrm{pH} \mathrm{10.4;} 10 \%(\mathrm{v} / \mathrm{v})$ methanol) and in cathode buffer were used. Transfer was carried out for $30-60 \mathrm{~min}$, at a current of $1.5 \mathrm{~mA} / \mathrm{cm}^{2}$. The membranes were treated with the antisera (purchased from Agrisera, Sweden) solutions, the resulting bands visualized by ECL (Amersham) and signals were recorded on X-ray film (Kodak). Stripping of the antibodies in order to probe one blot with different antibodies was carried out as recommended by the manufacturer of the ECL kit.

\section{Mass spectroscopy}

The in-gel digested samples were analyzed by ESI LCMS/MS using an HCT ultra ETD II iontrap instrument (Bruker) linked to an Easy nano LC system (Proxeon).
Processing, deconvolution, and compound detection for the LC-MS/MS datasets were performed using the Data Analysis software (4.0 SP4, Bruker). Database searches using the peak lists files of the processed datasets were performed using an in-house license of the Mascot search engine (Matrix science) and the current version of the Uniprot database (2012_01). The search parameters permitted a mass error of $0.3 \mathrm{Da}$ for both the MS and the MS/ MS mode and variable modifications of methionine by oxidation, of cysteine by propionamide derivation and $\mathrm{N}$-terminal acetylation.

\section{Oxygen evolution}

Oxygen evolution was assessed with a Clark-type electrode (Hansatech, England) at $20{ }^{\circ} \mathrm{C}$ in gel filtration buffer with $1 \mathrm{mM}$ 2,6-dichloro-p-benzoquinone, and $1 \mathrm{mM}$ ferricyanide as electron acceptors in the reaction mixture.

Acknowledgments This work was done with support from the Marie Curie program "Transfer of Knowledge" (MTKD-CT-2006042486), the Marie Curie program "European Reintegration Grant" (PERG05-GA-2009-247789) and the program "FSE SARDEGNA 2007-2013, Legge Regionale 7 agosto 2007, n. 7, Promozione della ricerca scientifica e dell'innovazione tecnologica in Sardegna"; DP and DdS are grateful to the ESRF and the Partnership for Structural Biology (Grenoble, France) for continuous support; we thank the Wallenberg and the Kempe Foundations for support of the instrumentation and bioinformatics infrastructure of the Proteomics Facility at Umeå University.

Open Access This article is distributed under the terms of the Creative Commons Attribution License which permits any use, distribution, and reproduction in any medium, provided the original author(s) and the source are credited.

\section{References}

Bassi R, Marquardt J, Lavergne J (1995) Biochemical and functional properties of photosystem II in agranal membranes from maize mesophyll and bundle sheat chloroplast. Eur J Biochem 233: 708-719

Boekema EJ, Hankamer B, Bald D, Kruip J, Nield J, Boonstra AF, Barber J, Rogner M (1995) Supramolecular structure of the photosystem II complex from green plants and cyanobacteria. Proc Natl Acad Sci USA 92:175-179

Cardona T, Sedoud A, Cox N, Rutherford AW (2012) Charge separation in photosystem II: a comparative and evolutionary overview. Biochim Biophys Acta 1817:26-43

Danielsson R, Suorsa M, Paakkarinen V, Albertsson P, Styring S, Aro E, Mamedov F (2006) Dimeric and monomeric organization of photosystem II. J Biol Chem 281:14241-14249

Dekker JP, Boekema EJ (2005) Supramolecular organization of thylakoid membrane proteins in green plants. Biochim Biophys Acta 1706:12-39

Fey H, Piano D, Horn R, Fischer D, Schröder WP, Bock R, Büchel C (2008) Isolation of highly active photosystem II core complexes with a His-tagged Cyt b559 subunit from transplastomic tobacco plants. Biochim Biophys Acta 1777:1501-1509 
Horton P, Wentworth M, Ruban A (2005) Control of the light harvesting function of chloroplast membranes: the LHCIIaggregation model for non photochemical quenching. FEBS Lett 579:4201-4206

Horton P, Johnson MP, Perez-Bueno ML, Kiss AZ, Ruban AV (2008) Photosynthetic acclimation: does the dynamic structure and macroorganisation of photosystem II in higher plant grana membranes regulate light harvesting states? FEBS J 275:1069-1079

Ishikawa Y, Nakatani E, Hemni T, Ferjani A, Harada Y, Tamura N, Yamamoto Y (1999) Turnover of the aggregates and crosslinked products of the D1 protein generated by acceptor side photoinhibition of photosystem II. BBA Bioenerg 1413:147-158

Kereïche S, Kiss AZ, Kouril R, Boekema EJ, Horton P (2010) The PsbS protein controls the macro-organisation of photosystem II complexes in the grana membranes of higher plant chloroplasts. FEBS Lett 584:759-764

Kiss AZ, Ruban AV, Horton P (2008) The PsbS protein controls the organization of the photosystem II antenna in higher plant thylakoids membranes. J Biol Chem 283:3972-3978

Li XP, Bjorkman O, Shih C, Grossman AR, Rosenquist M, Jansson S, Niyogi KK (2000) A pigment-binding protein essential for regulation of photosynthetic light harvesting. Nature 403:391-395

Li X, Gilmore AM, Caffari S, Bassi R, Golan T, Kramer D, Niyogi KK (2004) Regulation of photosynthetic light harvesting involves intrathylakoid lumen $\mathrm{pH}$ sensing by the PsbS protein. J Biol Chem 279:22866-22874

Niyogi KK, Li X, Rosenberg V, Jung H (2005) Is PsbS the site of nonphotochemical quenching in photosynthesis? J Exp Bot 56(411): 375-382

Pagliano C, Chimirri F, Saracco G, Marsano F, Barber J (2011) Onestep isolation and biochemical characterization of highly active plant PSII monomeric core. Photosynth Res 108:33-46

Piano D, El Alaoui S, Korza HJ, Filipek R, Sabala I, Haniewicz P, Buechel C, De Sanctis D, Bochtler M (2010) Crystallization of the photosystem II core complex and its chlorophyll binding subunit CP43 from transplastomic plants of Nicotiana tabacum. Photosynth Res 106:221-226

Pokorska B, Zienkiewicz M, Powikrowska M, Drozak A, Romanowska E (2009) Differential turnover of the photosystem II reaction centre D1 protein in mesophyll and bundle sheath chloroplast of maize. Biochim Biophys Acta 1787:1161-1169

Porra RJ, Thompson WA, Kriedmann PE (1989) Determination of accurate extinction coefficients and simultaneous equations for assaying chlorophylls a and $\mathrm{b}$ with four different solvents: verifications of the concentration of chlorophyll standards by atomic absorption spectroscopy. Biochim Biophys Acta 975:384-394

Schägger H, Jagow GV (1987) Tricine sodium dodecyl-sulfate polyacrylamide-gel electrophoresis for the separation of proteins in the range from 1 to $100-\mathrm{kDa}$. Anal Biochem 166:368-379

Schägger H, Jagow GV (1991) Blue native electrophoresis for isolation of membrane protein complexes in enzymatically active form. Anal Biochem 199:223-231

Szabó I, Bergantino E, Giacometti GM (2005) Light and oxygenic photosynthesis: energy dissipation as a protection mechanism against photo-oxidation. EMBO Rep 6:629-634

Takahashi T, Inoue-Kashino N, Ozawa S, Takahashi Y, Kashino Y, Satoh K (2009) Photosystem II complex in vivo is a monomer. J Biol Chem 284:15598-15606

Teardo E, Polverino de Laureto P, Bergantino E, Dalla Vecchia F, Rigoni F, Szabó I, Giacometti GM (2007) Evidences for interaction of PsbS with photosynthetic complexes in maize thylakoids. Biochim Biophys Acta 1767:703-711

Watanabe M, Iwai M, Narikawa R, Ikeuchi M (2009) Is the photosystem II complex a monomer or a dimer? Plant Cell Physiol 50(9):1674-1680

Yi X, McChargue M, Laborde S, Frankel LK, Bricker TM (2005) The manganese-stabilizing protein is required for photosystem II assembly/stability and photoautotrophy in higher plants. J Biol Chem 280(16):16170-16174 